\title{
Correction to: Incentive mechanisms for mobile crowd sensing based on supply-demand relationship
}

\author{
Jia Xu ${ }^{1} \cdot$ Wei Lu ${ }^{1} \cdot$ Lijie Xu ${ }^{1}$ Dejun Yang ${ }^{2} \cdot$ Tao Li $^{1}$
}

Published online: 25 November 2019

(C) Springer Science+Business Media, LLC, part of Springer Nature 2019

Correction to: Peer-to-Peer Netw. Appl. 2019, 12(3): 577-588 https://doi.org/10.1007/s12083-018-0648-y

Correction is needed in the original article.

The university name in affiliation (1) is changed from "University of Posts and Telecommunications" to "Nanjing University of Posts and Telecommunications".

The correct affiliation of authors excepting Dejun Yang is "Jiangsu Key Laboratory of Big Data Security \& Intelligent Processing, Nanjing University of Posts and Telecommunications, Nanjing, China.

The authors apologize for this mistake.

The online version of the original article can be found at https://doi.org/ 10.1007/s12083-018-0648-y

Jia Xu

xujia@njupt.edu.cn

1 Jiangsu Key Laboratory of Big Data Security \& Intelligent Processing, Nanjing University of Posts and Telecommunications, Nanjing, China

2 Department of Computer Science, Colorado School of Mines, Golden, CO, USA 